Please cite as: R.G. Harrison et al, Planetary Atmospheric Electricity, Space Sci. Rev 137, 14, 5-10 (2008) doi: 10.1007/s11214-008-9397-1

\title{
Planetary atmospheric electricity
}

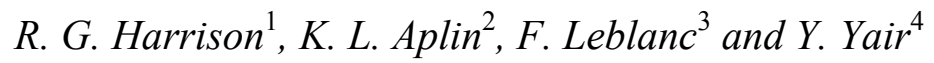

\begin{abstract}
Electrification is a fundamental property of planetary atmospheres, found widely in the solar system. It is most evident through lightning discharges, which can influence an atmosphere's chemical composition, but electrification also affects the physical behaviour of aerosols and cloud droplets that determine an atmosphere's radiative balance. In the terrestrial atmosphere, lightning has been implicated in the origin of life.
\end{abstract}

${ }^{1}$ Department of Meteorology, University of Reading, Earley Gate, Reading, UK email: r.g.harrison@reading.ac.uk

${ }^{2}$ Space Science and Technology Department, Rutherford Appleton Laboratory, Didcot, Oxon, UK

${ }^{3}$ Service d'Aeronomie du CNRS (at Osservatorio Astronomico di Trieste, 34131

Trieste, Italy)

${ }^{4}$ Department of Life and Natural Sciences, Open University of Israel, Ra'anana 43107 Israel 


\section{Electrification in atmospheres}

Electrification occurs commonly in planetary atmospheres, although it is present in different forms. The most direct evidence to remote observers is presented by lightning, which is definitively known to occur on Earth, Jupiter, Saturn and, most probably, Venus, with lightning on Mars thought very likely but as yet undetected. In addition, Uranus and Neptune have yielded possible signals of lightning discharges. Taken across the solar system, the probability of lightning occurring in a planetary atmosphere (Venus, Earth, Mars, Jupiter, Saturn, Uranus and Neptune, but neglecting moons) is therefore confidently at least $(4 \pm 1)$ in 7 . Thus, as lightning can also result from volcanic activity, it is reasonable to expect electrification in a solar system atmosphere, with odds considerably better than evens.

Planetary electrification is generally explained by analogy with terrestrial processes. Lightning on both Saturn and Jupiter is thought to originate from water clouds deep in their atmospheres, with temperatures $\sim 300 \mathrm{~K}$ (Fischer et al. 2008) where water is likely to exist in more than one phase. Charge separation is therefore expected to develop in a similar way to terrestrial thunderclouds (Saunders 2008). Martian lightning, though not yet measured, is likely to originate in dust storms. These storms are probably directly analogous to terrestrial dust devils, in which high electric fields are generated by triboelectric charge separation ${ }^{1}$ (Farrell and Desch 2001). There is, however, no terrestrial analogue for the sulphuric acid clouds on Venus. Observations of Venus lightning are increasingly convincing (e.g. Russell et al. 2007), but a scientific consensus on the existence of Venusian lightning has been slow to develop, probably because of the clear differences between Venusian and other solar system lightning (Gurnett et al. 2001). This illustrates that much of our acceptance of planetary atmospheric electricity is rooted in comparison with terrestrial models.

Lightning highlights atmospheric electrification localised in space and time. Charge released by high-energy particles, such as Galactic Cosmic Rays (GCR) is, however, spatially and temporally widespread in an atmosphere. The incessant GCR flux sweeping across the solar system from stellar and galactic sources ensures that planetary atmospheres are constantly permeated by energetic particles. Their arrival rate is modulated by solar activity (with the 11-year Schwabe cycle), though this effect decreases with distance from the Sun, and by the planetary magnetic field (Bazilevskaya et al. 2008). Together with the ultraviolet part of the solar spectrum, GCRs constitute energetic ionisation agents, although the depth of penetration and the nature of ionisation products are specific to each planetary atmosphere.

\section{Ion production, clouds and atmospheric discharges}

As well as GCR ionisation, rocky planetary bodies have a low-altitude ionisation source from the radioactive minerals contained within the surface rocks, whose decay products emanate and ionise the air immediately above it. Charging of aerosols generated mechanically from the planet's surface is expected to be a direct consequence. In the presence of condensable compounds, varying in composition between atmospheres, ion production may affect the nucleation of ultra-fine aerosol particles (Arnold 2008, Kazil et al. 2008) or droplet condensation, thus providing a

\footnotetext{
${ }^{1}$ On Earth, however, the breakdown voltage is sufficiently large that discharges in dust devils generally do not occur.
} 
link between ionisation and cloud formation. The connection between ions and clouds is especially important in Earth's atmosphere because of the central role clouds play in the climate system, now studied more intensively than ever because of the challenging context presented by climate change.

The presence of ionisation in cloud-forming atmospheric regions indicates that charging can affect many microphysical processes in clouds (e.g. ion-droplet attachment, droplet activation, droplet coalescence and aerosol-droplet scavenging) which determine cloud lifetime, cloud thickness and ultimately precipitation, and also the complex charging processes leading to electric field growth. Cloud microphysical processes and charging occur simultaneously as thunderclouds mature, and are intricately inter-related. Modern theories suggest that GCR-induced free electrons within a developing thundercloud are actually responsible for triggering the breakdown process, which culminates in an avalanche, propagating between charge centres or to the ground as a lightning flash. Electrons and ions also exist above cloud tops, where they are subjected to transient electric fields following lightning discharges; acceleration of electrons and ions upwards above terrestrial cloud tops creates spectacular transient luminous events (TLEs), known as sprites and elves. Terrestrial gamma flashes (TGFs) may also result from lightning's aftermath.

When a lightning channel passes through an atmosphere $\left(\mathrm{N}_{2}\right.$ or $\mathrm{CO}_{2}$ based), it initiates chains of chemical reactions whose long-lived products can be remotely detected, permitting elucidation of the atmospheric composition. On Earth, $\mathrm{NO}_{\mathrm{x}}$ from lightning is transported over large distances in the anvils of thunderstorms; the chemical effects are prolonged, ultimately providing a source of dissolved solutions at the surface.

\section{Evidence for planetary atmospheric electrification}

Non-terrestrial electricity in upper atmospheres has generally been studied by ion and electron spectrometers, electric and magnetic field measurements and plasma wave instruments, as well as remotely, from earth, by radio-occultation, optical spectrometry, electromagnetic and radar measurements (Aplin et al. 2008). Upper atmosphere measurements characterise the ionospheres produced by energetic ultraviolet ionisation but, in some cases, a secondary peak in electron concentration due to meteors has been discovered, below the ionospheric electron region (Molina-Cuberos et al. 2008).

Considering lower atmospheres, no definitive evidence for lightning has yet emerged from the Huygens descent through the atmosphere of Saturn's satellite Titan. No Martian lightning activity has ever been measured but, so far, no electrical instrument has deployed at its surface and therefore scope for its detection remains. Very recently, Venus Express magnetic measurements have strongly suggested lightning on Venus, although without a coincident optical observation, a small ambiguity over the precise source remains. Ground-based observations of NO abundance in the Venusian atmosphere also suggest lightning activity (Krasnopolsky 2006). Voyager 1 first detected optical lightning and the electromagnetic signatures of whistlers ${ }^{2}$ at Jupiter. It subsequently detected sferics $^{3}$ and whistlers at Saturn, a discovery recently

\footnotetext{
${ }^{2}$ Whistlers are radio signals below $f \sim 30 \mathrm{kHz}$. In physical terms they propagate in the frequency interval between the ion and electron cyclotron frequencies $f_{\mathrm{ci}}<<f<f_{\mathrm{ce}}$.

${ }^{3}$ Sferics (a word derived originally from "atmospherics" in radio work) describes high frequency electromagnetic emissions.
} 
confirmed by the Cassini mission (Fischer et al., 2008). Voyager 2 measured sferics at Neptune and Uranus, interpreted as the signatures of lightning activity (Yair et al. 2008, Zarka et al. 2008).

As an alternative to direct optical detection (Takahashi et al. 2008), electromagnetic emissions have assumed to present clear signatures of lightning activity in planetary atmospheres (Zarka et al. 2008). However recent work arising from terrestrial atmospheric electricity has suggested some new approaches to studying planetary atmospheric electricity. Very low frequency measurements $(3 \mathrm{~Hz}-3 \mathrm{kHz})$ can indicate the Schumann resonance, which is caused by lightning and its presence presents an important clue to the existence of a global circuit (Aplin et al. 2008, Simoes et al. 2008). The uppermost ultra low frequency present in the spectrum may be associated with lightning activity (Bosinger et al. 2008). Transient luminous events discovered above active thunderstorms provide a further possibility for characterising lightning activity optically, even on planets completely covered by clouds (Yair et al. 2008). Terrestrial Gamma and X-Ray flashes associated with intra-cloud discharges are other potentially detectable phenomena which may occur more widely in the solar system (Lefeuvre et al. 2008, Roussel-Dupré et al. 2008).

\section{Atmospheric current flow and global electrical circuits}

The balance between charge generation, lightning and local ionisation results, on earth at least, in a planetary scale current flow between disturbed and fair weather regions. More generally, this conceptual circuit model provides a framework unifying atmospheric electrical processes operating in disturbed weather regions (e.g. thunderclouds, dust devils, volcanic plumes) and the ionisation processes occurring throughout the atmosphere. Conceived by CTR Wilson, the terrestrial global circuit model is based on the generation of a global potential difference between conducting upper and lower regions, separated by a poorly conducting atmosphere (Rycroft et al. 2008). This potential difference drives a current of cluster ions (and/or electrons) created by cosmic rays and natural radioactivity between the two conducting layers. Through the global circuit current, the source energy from thunderstorms is globally dissipated by the transport of trace species, and a steady flow of ions is maintained throughout the widespread non-thunderstorm regions.

As the study of terrestrial atmospheric electricity is long established, there is much that can be learnt from its history (Aplin et al. 2008). Understanding the terrestrial concepts, and synthesising them in to produce the global circuit model was protracted, taking two centuries. Identifying the key stages in the synthesis could be used to optimise future measurements of extraterrestrial environments. For example, the Schumann resonance (low frequency radiation caused by the lightning excitation of the cavity formed between the atmosphere's lower and upper conducting layers) is one of the more recently discovered aspects of the terrestrial global circuit (1950s). It has been identified as the most informative single measurement to make (Aplin et al. 2008), and therefore deserves priority when proposing future planetary atmospheric electrical instrumentation.

\section{Significance of atmospheric electrification}

Clearly, our knowledge of terrestrial lightning can be applied to mitigating electrostatic hazards. This is put to very practical application when designing lightning protection systems for spacecraft. All spacecraft are under threat from 
lightning after launch (or sometimes before (Lorenz 2008)), particularly because the tropical locations where spacecraft are launched are also where lightning is most common. Planetary probes need additional protection against electrostatic discharge (ESD) hazards when passing through atmospheres or landing on other planets. For the Huygens and Galileo probes, ESD technology was directly borrowed from the aircraft industry (Lorenz 2008).

There are, however, two broader scientific issues extending well beyond the essential protection of spacecraft. Firstly, fossil evidence confirms that terrestrial lightning has existed for at least 250 million years (Harland and Hacker 1966), so, as well as being abundant in the solar system, electrification is probably a long-lived phenomenon on geological timescales. The abundance and longevity of terrestrial lightning has caused it to be suggested as a possible factor in the formation of molecules central to the origin of life (Miller 1953). Secondly, as outlined above, charge can modify cloud formation processes and the collision rates between droplets, crystals and aerosols. Although the effect of electrification on individual aerosols and droplets may be small, it can also be widespread, acting, in total, to influence an atmosphere's radiative balance ${ }^{4}$.

Beyond lightning in planetary atmospheres, aspects in their infancy include the role of electrical forces in lifting dust (Renno et al. 2008), as well as the importance of cluster ions and charged aerosols (Tripathi et al. 2008, Harrison and Tammet 2008, Aplin 2008), Some further aspects of non-terrestrial planetary electricity have also hardly been characterised, such as the charging of planetary rings (Graps et al. 2008), volcanic electrical activity on Io (James et al. 2008) and the charge carried by asteroids.

\section{Conclusions}

Atmospheric electricity can originate from many different causes, specific to the kind of atmosphere and the altitude above the planetary surface. In all gaseous planetary atmospheres charge is generated by cosmic rays or ultraviolet radiation, but also by friction, meteoric impacts, atmospheric circulation, cloud charging, volcanism, and dust. The presence of aerosols modifies the charging process, facilitating charge transfer between ions and aerosols. In other planetary environments, dust storms or impacts are responsible for charge production. Rings around planets can become charged both actively and passively - for instance in the presence of magnetic fields and plasma - with their dynamics being at least affected, if not completely determined, by the built up electric fields. In one or the other of these ways, charged atmospheric layers can be produced near most of the planets.

Information about planetary atmospheric electricity has been obtained by spacecraft observations, and the extraordinarily valuable measurements made in situ when spacecraft pass through a planetary atmosphere or even land instrumentation on the planet. On Earth, the violent discharge from large atmospheric electric fields is common and evident through lightning. On other planets, however most discharges manifest themselves in secondary effects, which, from lightning, is frequently the non-optical electromagnetic radiation that can leak from their atmospheres.

\footnotetext{
${ }^{4}$ This was memorably described at the EuroPlanet-ISSI workshop in Bern as "the tail wagging the dog".
} 
Consequently detailed study of terrestrial lightning remains very important, and therefore many of the articles in this book are in one or the other way concerned with lightning, its causes, mechanism, effects, intracloud lightning, cloud to ground lightning, and cloud to space lightning like TLEs, as well as the generation of electromagnetic radiation from Schumann resonances through the spectrum to X-rays, and TGFs. The latter two are signatures of high-energy particles generated in the lightning discharges; they may indicate planetary atmospheric electric fields which could present hazards for missions to the planets.

In this volume (Leblanc et al 2008), a detailed and up to date summary is given of the various problems in planetary atmospheric electricity outlined above. A feature is that it begins with an introductory overview section. These introductory overviews are intended to provide a brief, though fairly-founded, tutorial concerning the physics and chemistry of atmospheric electricity on Earth and the planets, the charging, ion production, current flow, current systems, electrical conductivities, and the observable processes involved into the quiet and the violent discharges. Based on these articles, the student and researcher of the many and various forms of atmospheric electricity should be prepared to deal with the more specialised papers that follow. 
Please cite as: R.G. Harrison et al, Planetary Atmospheric Electricity, Space Sci. Rev 137, 14, 5-10 (2008) doi: 10.1007/s11214-008-9397-1

\section{References}

Aplin K, Composition and measurement of charged atmospheric clusters, this volume (2008)

Aplin K, M Rycroft and G Harrison, Investigation of Earth's atmospheric electricity: a role model for planetary studies, this volume (2008)

Arnold F, Atmospheric ions and aerosol formation, this volume (2008)

Bazilevskaya G A, I G Usoskin, E Flückiger et al, Cosmic ray induced ion production in terrestrial and planetary atmospheres, this volume (2008)

Bosinger and Shalimov, On ULF signatures of lightning discharges (2008)

Russell C T, T L Zhang, M Delva et al, Nature 450, 661-662, doi:10.1038/nature05930 (2007)

Gurnett D A, P Zarka, R Manning et al, Nature 409, 313-315 (2001)

Fischer G, D A Gurnett, W S Kurth et al, Atmospheric electricity at Saturn, this volume (2008)

Graps A, G Jones, A Jubasz et al, Charging of planetary rings, this volume (2008)

Harrison $\mathrm{G}$ and $\mathrm{H}$ Tammet, Ions in the terrestrial atmosphere and other solar systems atmospheres, this volume (2008)

Harland W B and J L F Hacker, "Fossil” lightning strikes 250 million years ago, Adv Sci 22, 663-671 (1966)

James M R, L Wilson, S J Lane et al, Electrical charging of volcanic plumes, this volume (2008)

Kazil J, R G Harrison and E R Lovejoy, Tropospheric new particle formation and the role of ions, this volume (2008)

Krasnopolsky V A, Chemical composition of Venus atmosphere and clouds: Some unsolved problems, Plan Space Sci 54, 13-14, 1352-1359 (2006)

Leblanc F, Aplin K L, Yair Y et al, Planetary Atmospheric Electricity, Space Sciences Series of ISSI, 30 (2008)

Lefeuvre F, E Blanc, R Roussel-Dupré et al, Taranis - a microsatellite project dedicated to the physics of TLEs and TGFs, this volume (2008)

Lorenz R, Atmospheric electricity hazards, this volume (2008)

Molina-Cuberos G, J J Lopez-Moreno and F Arnold, Meteoritic ions in planetary atmospheres, this volume (2008)

Miller S L, Production of Amino Acids Under Possible Primitive Earth Conditions, Science 117528. doi:10.1126/science.117.3046.528 (1953)

Renno N and J Kok, Electric activity and dust lifting on Earth, Mars and beyond, this volume (2008)

Roussel-Dupré R, J J Colman, E Symbolisty et al, Physical processes related to discharges in planetary atmospheres, this volume (2008)

Rycroft M, R G Harrison, K A Nicoll et al, Physical processes related to discharges in planetary

atmospheres, this volume (2008)

Saunders C, Charge separation mechanisms in clouds, this volume (2008)

Simoes F, M Rycroft, N Renno et al, Schumann resonances as a means of investigating the electromagnetic environment in the solar system, this volume (2008)

Takahashi Y, J Yoshida, Y Yair et al, Lightning detection by LAC onboard the Japanese Venus

Climate Orbiter Planet-C, this volume (2008)

Tripathi S, M Michael and R G Harrison, Profiles of ion and aerosol interactions in planetary atmospheres, this volume (2008)

Farrell W M, M D Desch, J Geophys Res E4, 7591-7595 (2001)

Yair Y, G Fischer, F Simoes et al, Updated review of planetary atmospheric electricity, this volume (2008)

Zarka P, W Farrell, G Fischer et al, Ground-based and space-based radio observations of planetary lightning, this volume (2008) 